\title{
Recul des glaciers de montagne : que nous apprennent les satellites?
}

\author{
Étienne Berthier ${ }^{(1,2)}$ \\ (1) Université de Toulouse, UPS (OMP-PCA) - Legos \\ 14, avenue Édouard-Belin - 31400 Toulouse \\ (2) CNRS, OMP-Legos
}

\section{Résumé}

Les glaciers de montagne constituent l'un des témoins les plus spectaculaires de l'évolution climatique récente et contribuent significativement à l'élévation actuelle du niveau moyen des mers. Paradoxalement, seul un nombre réduit de glaciers est étudié in situ (environ cinquante), car ils sont disséminés à la surface de la Terre et restent difficiles d'accès. Depuis quelques années, l'imagerie satellitaire est utilisée par les glaciologues pour dresser un constat alarmant de leur évolution récente.

\section{Abstract}

The retreat of mountain glaciers: what can satellites tell us?

Mountain glaciers are one of the best indicators of climate change and their rapid wastage make them a strong contributor to sea level rise. The estimated 160,000 mountain glaciers are spread all around the globe and remain difficult to access. Consequently, only a limited number (about 50 glaciers) are regularly monitored in the field. Today, high resolution satellite optical images are combined to some advanced methodologies to survey their fast and alarming evolution.
$\mathrm{C}$ article traite de l'observation atellitaire de l'évolution récente des glaciers de montagne et des petites calottes glaciaires en réponse aux changements climatiques. Depuis l'article fondateur de Meier (1984), ces entités de la cryosphère sont étudiées conjointement (et donc séparément des deux calottes polaires antarctique et groenlandaise) car elles mettent en jeu des échelles spatiales voisines (quelques kilomètres à quelques dizaines de kilomètres) et donc des méthodes d'étude souvent similaires. Ainsi, le plus grand « glacier» (terme qui, dans la suite de cet article, désigne à la fois les glaciers de montagne et les petites calottes glaciaires) est la calotte du nord de l'île d'Ellesmere qui couvre $26000 \mathrm{~km}^{2}$, soit soixante-cinq fois moins que la calotte groenlandaise et cinq cents fois moins que la calotte antarctique.

\section{Témoins \\ des changements climatiques et acteurs des bilans hydrologiques}

Les glaciers de montagne ont une taille variable et sont nichés au cœur d'une topographie abrupte. Leur volume total équivaut à environ $0,3 \mathrm{~m}$ de niveau marin. Ils se rencontrent à toutes les latitudes du globe, mais c'est aux hautes latitudes que l'on trouve les plus vastes étendues glaciaires comme les champs de glace (icefield en anglais) d'Alaska et de Patagonie. Les glaciers d'Asie centrale sont une exception à cette règle, occupant à eux seuls plus de $115000 \mathrm{~km}^{2}$ entre $28^{\circ} \mathrm{N}$ et $42^{\circ} \mathrm{N}$.
Les petites calottes glaciaires (ice cap en anglais par opposition aux deux ice sheet ou calottes polaires) constituent des modèles réduits des calottes polaires : elles créent leur propre topographie, ont une région centrale faiblement pentue alors qu'en périphérie la glace est évacuée par de rapides glaciers de vallée. Une calotte glaciaire couvre en général plusieurs milliers de kilomètres carrés (par exemple, $8000 \mathrm{~km}^{2}$ pour la calotte du Vatnajökull en Islande, soit la superficie de la Corse). Leur difficulté d'accès, leur éloignement des zones habitées et l'intérêt que suscitent les deux calottes polaires antarctique et groenlandaise font qu'il s'agit de la composante la moins étudiée de la cryosphère. Pourtant, elles contiendraient (au moins) la même quantité d'eau que les glaciers de montagne (Dyurgerov et Meier, 2005). Leur localisation dans des régions où le réchauffement climatique est sensible (Arctique, péninsule Antarctique) conforte l'idée qu'elles doivent être plus amplement étudiées.

L'eau douce stockée dans les glaciers (de montagne et petites calottes) équivaut à $0,65 \mathrm{~m}$ de niveau marin (Dyurgerov et Meier, 2005), ce qui est faible par rapport à la calotte groenlandaise $(7 \mathrm{~m})$ ou antarctique $(65$ à $70 \mathrm{~m})$. Toutefois, les glaciers répondent rapidement aux fluctuations du climat et, donc, aux courtes échelles de temps, influencent les bilans hydrologiques locaux, régionaux et globaux. Par exemple, ils perdent rapidement de la masse depuis les années 1980 et participent à la hausse du niveau marin, alors que leur surface cumulée dépasse à peine celle de la France. Entre 1993 et 2003, ils ont contribué au tiers (soit environ $1 \mathrm{~mm} / \mathrm{an}$ ) de la hausse totale du niveau moyen des mers qui atteint 3 mm/an (IPCC, 2007). Entre 
2003 et 2008 , leur contribution approche même $50 \%$ (Cazenave et al., sous presse). Ces flux additionnels d'eau douce vers l'océan diminuent la salinité, donc la densité des masses d'eau superficielles, ce qui pourrait altérer la circulation océanique, notamment dans les régions arctiques. Plus localement, les glaciers jouent également un rôle régulateur du régime des rivières en stockant l'eau à l'état solide durant la saison humide et en la restituant sous forme liquide durant la saison sèche (Francou et Vincent, 2007). Ils soutiennent ainsi les débits d'étiages des cours d'eau et leur présence est parfois cruciale pour la ressource en eau des populations des vallées de montagne, voire de certaines capitales d'Amérique du Sud ou d'Asie du Sud-Est : irrigation des cultures, microcentrales électriques, consommation domestique. Mais pendant combien de temps les glaciers assureront-ils encore ce rôle de château d'eau naturel?

Enfin, l'état de santé des glaciers de montagne, présents à toutes les latitudes et à diverses altitudes, constitue un excellent indicateur des fluctuations climatiques (Oerlemans, 2005 ; Vincent at al., 2004 ; Ohmura et al., 2007). Ils sont révélateurs des évolutions des températures et des précipitations dans des régions où les mesures météorologiques sont rares et peu homogènes. Situés le plus souvent dans des régions sous faible pression anthropique, ils témoignent de l'évolution d'un climat non influencé par des phénomènes locaux comme la croissance urbaine ou l'utilisation des sols. Autant d'enjeux qui invitent à un suivi attentif de ces témoins du réchauffement global.

Toutefois, notre connaissance de l'évolution et du fonctionnement des glaciers demeure fragmentaire et limitée par le manque d'observations. Les mesures in situ sont rendues délicates par les conditions climatiques propres à l'environnement de haute montagne (tempêtes de neige, froid, crevasses nombreuses, risques d'avalanche). Aussi, parmi quelque 160000 glaciers disséminés à la surface du globe (ce chiffre est une estimation car il n'existe pas encore d'inventaire complet des glaciers), seule une cinquantaine (de petite taille et les plus faciles d'accès) est régulièrement visitée sur le terrain par les glaciologues (Braithwaite, 2002). Pour obtenir des observations à l'échelle globale et pouvoir étudier les plus grands glaciers (qui sont aussi les plus gros contributeurs potentiels à l'élévation du niveau marin, voir Arendt et al., 2002), l'observation satellitaire semble évidemment un outil adapté. Mais son utilisation est rendue difficile par la grande hétérogénéité de taille des objets étudiés (de quelques centaines de $\mathrm{m}^{2}$ à quelques milliers de $\mathrm{km}^{2}$ ) et la forte topographie des zones de montagne. Par exemple, il n'est pas possible d'utiliser l'altimétrie radar qui a fait largement ses preuves pour l'étude des calottes polaires antarctique et groenlandaise (Rémy et al., 2000). L'empreinte au sol d'un altimètre est de plusieurs kilomètres, soit plus que la largeur de la majorité des glaciers de montagne ! Une autre difficulté émane d'un état de surface qui évolue très rapidement (à l'échelle de quelques jours) en raison des chutes de neige ou de la fonte. Du coup, la perte de cohérence (ou de corrélation) est fréquente lorsque l'on compare deux acquisitions satellitaires, notamment radar (Fischer et al., 2003). Enfin, les glaciers, couverts de neige ou de glace, ont un fort albédo et réfléchissent donc la plus grande partie du rayonnement solaire vers l'espace, ce qui sature les images si les gains instrumentaux ne sont pas ajustés au préalable. Les difficultés sont donc nombreuses, mais peuvent être levées par une définition minutieuse des conditions géométriques et radiométriques des acquisitions satellitaires et par des posttraitements adaptés au contexte de haute montagne. Aujourd'hui, des images optiques haute résolution nous permettent de mesurer deux variables clés des glaciers : leurs vitesses de surface et leurs changements de volume.

Dans cet article, nous présenterons rapidement les méthodes qui permettent ces mesures depuis l'espace, mais également leur validation. À ce titre, les glaciers du massif du Mont-Blanc, régulièrement visités par les glaciologues (Vincent et al., 2007b), constituent une cible de choix pour confronter nos méthodologies avec les campagnes de terrain du Laboratoire de glaciologie et géophysique de l'environnement de Grenoble [LGGE]. Nous verrons aussi comment ces méthodes peuvent être appliquées à des sites plus difficiles d'accès comme les calottes glaciaires islandaises ou des Kerguelen, les glaciers de l'Himalaya indien ou ceux du Spitzberg. Nous conclurons sur l'apport de ces techniques pour l'étude des glaciers émissaires des calottes antarctique et groenlandaise dans le cadre de la quatrième année polaire internationale.

\section{Évolution des surfaces et volumes glaciaires}

Il est relativement aisé de mesurer les changements de longueur et de surface d'un glacier depuis l'espace. Au moins deux images, généralement espacées de quelques années ou quelques dizaines d'années, sont recalées le plus

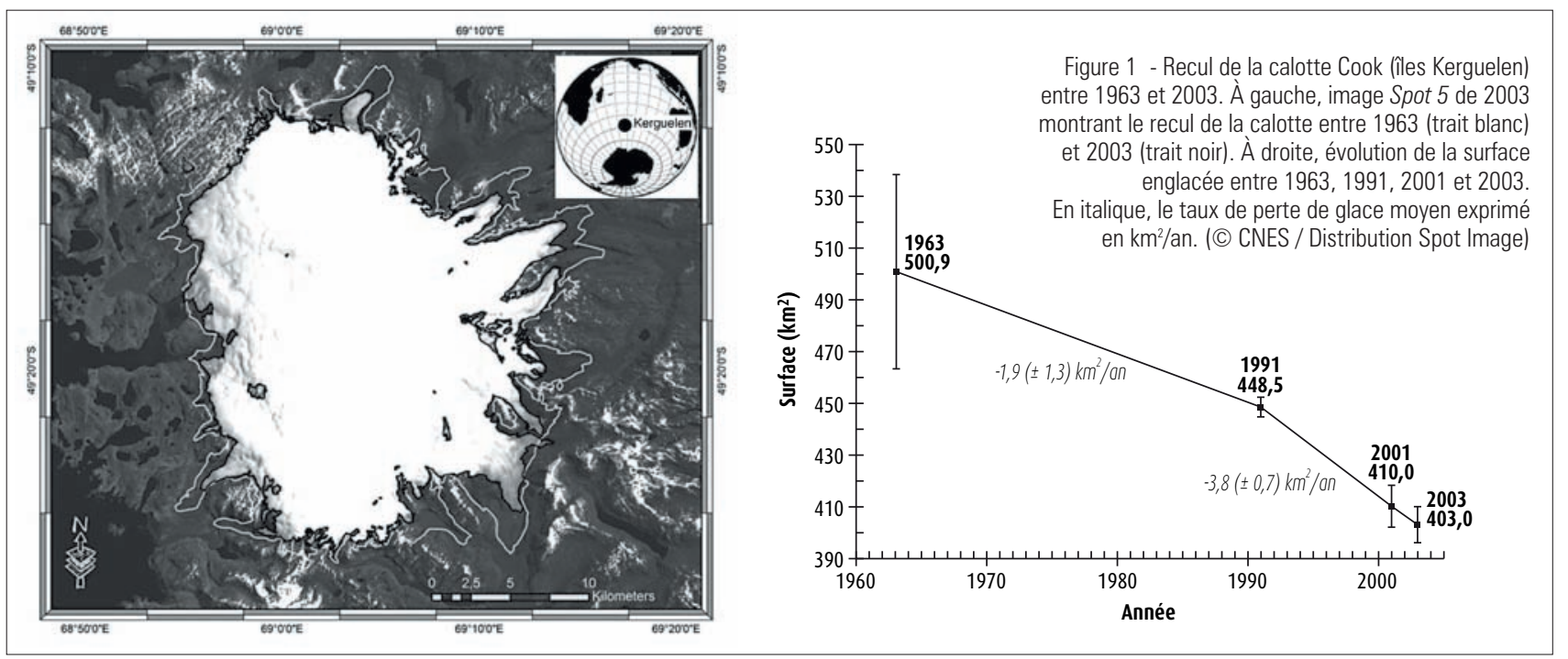


précisément possible et les limites du glacier sont alors digitalisées. Cette dernière opération peut être réalisée manuellement (ce qui est laborieux mais reste la technique la plus fiable) ou automatiquement grâce à des algorithmes qui distinguent (tant bien que mal) la signature spectrale de la glace et de la neige de celle des roches, de la végétation ou plus difficilement des nuages (Paul et al., 2002). Des

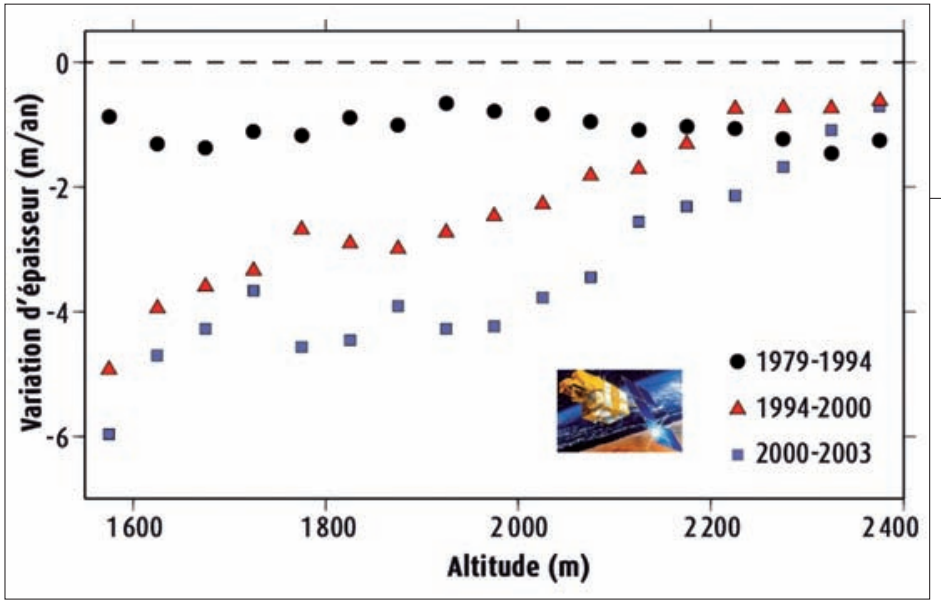

Figure 2 - Variations d'épaisseur de la partie basse

de la mer de Glace en fonction de l'altitude pour trois périodes 1979-1994; 1994-2000 et 2000-2003.

confirme que la relation entre variations glaciaires et changement climatique n'est pas simple. Plus loin dans cet article, nous reviendrons sur la moitié non expliquée des pertes d'épaisseur.

Outre la mer de Glace, images satellitaires récentes peuvent être comparées, non seulement entre elles, mais aussi à des cartes plus anciennes pour détecter des évolutions sur le long terme. À titre d'exemple, c'est ce que nous venons de faire au Laboratoire d'études en géophysique et océanographie spatiales [Legos], afin de documenter l'évolution des surfaces glaciaires des îles Kerguelen dans la partie australe de l'océan Indien. Sur ces îles difficiles d'accès, des observations glaciologiques ont été réalisées dans les années 1970 lors de campagnes de terrain (Vallon, 1977) et depuis... plus rien ! Entre 1963 (date de la cartographie au $1 / 200000$ de l'île par l'IGN) et 2003 (date d'acquisition d'une image par le satellite Spot 5), la surface de la calotte Cook (la principale masse de glace des îles Kerguelen) est passée de 501 à $403 \mathrm{~km}^{2}$, une diminution spectaculaire de $20 \%$ (figure 1). Grâce à une autre image Spot de 1991, nous montrons également que ce recul est deux fois plus rapide entre 1991 et 2003 qu'au cours de la période 1965-1991. Le retrait glaciaire est encore plus important pour les autres glaciers des Kerguelen, certains étant sur le point de disparaître.

Mais plus que les variations des surfaces couvertes par les glaciers, ce sont les changements des volumes glaciaires (autrement dit, leur bilan de masse global) qui confèrent aux glaciers le titre d'indicateur climatique et permettent d'évaluer leur contribution à l'élévation du niveau moyen des mers. Mais cette mesure est beaucoup plus délicate, notamment sur des périodes de temps de quelques années à quelques décennies : il ne s'agit plus cette fois de détecter un recul qui atteint plusieurs centaines de mètres, mais des variations de l'altitude de la surface du glacier (épaississement ou amincissement) de l'ordre de plusieurs mètres, voire quelques dizaines de mètres pour les évolutions les plus rapides. Pour ce faire, des topographies précises, déduites de couples stéréoscopiques d'images Spot pour des années différentes, sont comparées pour estimer les variations d'épaisseur de glace. Au préaces différents modèles numériques de terrain (MNT, représentation numérique de la topographie) sur les zones rocheuses et stables qui entourent les glaciers. En effet, dans ces zones montagneuses où les pentes sont fortes, tout décalage horizontal des topographies pourrait induire des erreurs sur la mesure des changements d'altitude. Nos mesures dans le massif du Mont-Blanc, couvrant les périodes 1979-1994, 1994-2000 et 2000-2003, ont pu être comparées aux relevés in situ du LGGE (précis à $\pm 30 \mathrm{~cm}$ ), indiquant une précision de \pm 1 à $2 \mathrm{~m}$ pour la méthode satellitaire. Pour la première fois, on démontre que des variations d'épaisseur des glaciers de montagne peuvent être observées depuis l'espace (Berthier et al., 2004). Grâce à ces observations, une accélération nette de l'amincissement de la mer de Glace à basse altitude est observée lors des dix dernières années (figure 2) alors qu'aucune tendance significative n'apparaît au-dessus de 3000 m. Il est vrai que la méthode satellitaire est moins précise dans cette zone d'accumulation (enneigée même en plein été) où les images optiques manquent de contraste.

Ainsi, en face de la gare du Montenvers (arrivée du petit train qui monte depuis Chamonix) vers $1600 \mathrm{~m}$, la mer de Glace perd chaque année 3 à $4 \mathrm{~m}$ de glace et il faut ajouter des échelles pour que les alpinistes et les touristes puissent y prendre pied. Pour ce même glacier, nous avons constaté que le simple accroissement de la fonte en surface du glacier, liée à l'augmentation des températures, n'explique, grosso modo, qu'une moitié de ces fortes pertes d'épaisseur à basse altitude (travaux LGGE/Legos non publiés). C'est une constatation importante, car cela lable, il a fallu soigneusement ajuster nous avons cartographié les variations d'épaisseur de l'ensemble des glaciers du côté français du massif du MontBlanc entre 1979 et 2003 (figure 3). Comme pour la mer de Glace, l'amincissement est fort à basse altitude alors que les bassins d'accumulation résistent plutôt bien, ce qui est en accord avec une étude récente fondée sur des mesures in situ (Vincent et al., 2007a). Au cours des étés 2008 et 2009 , la réalisation d'une nouvelle topographie à partir d'images Spot 5 nous permettra de confirmer une éventuelle tendance à l'accélération des pertes d'épaisseur et surtout de cartographier ces pertes pour l'ensemble du massif du Mont-Blanc (en incluant les glaciers suisses et italiens). À l'avenir, la comparaison de cette topographie satellitaire avec la carte établie au début du $\mathrm{XX}^{\mathrm{c}}$ siècle par Henri et Joseph Vallot nous permettra également d'établir les variations cumulées des glaciers au cours du dernier siècle.

Notre méthode d'estimation des variations des volumes glaciaires est désormais bien validée et peut donc être appliquée aux glaciers plus difficiles d'accès et dont l'évolution reste très mal connue. C'est le cas notamment des glaciers de la chaîne himalayenne. Alors que la surface englacée himalayenne dépasse $30000 \mathrm{~km}^{2}\left(60000 \mathrm{~km}^{2}\right.$ si l'on ajoute le Karakorum et l'Hindu Kush), seulement quelques $\mathrm{km}^{2}$ de glace sont suivis sur le terrain chaque année. Des extrapolations (un peu hasardeuses) permettent tant bien que mal de conjecturer sur la contribution de ce massif à l'élévation du niveau des mers (Kaser et al., 2006). Il y a donc un manque criant d'observations glaciologiques dans cette région. Cette lacune est en partie comblée grâce à des campagnes de terrain annuelles, conduites par l'Institut de recherche pour le développement [IRD] (UR Great Ice) et qui permettent de mesurer le bilan de masse d'un glacier indien, le Chhota Shigri, depuis 2002 (Wagnon et al., 
Figure 3 - Variation d'épaisseur (en mètres) de différents glaciers du massif du Mont-Blanc entre 1979 et 2003. En gris sur la carte, les zones pour lesquelles aucune donnée n'est disponible. Les courbes montrent les variations d'épaisseur, glacier par glacier, moyennées par tranche d'altitude.

Les différents glaciers sont du nord au sud glacier du Tour (TOU), d'Argentière (ARG), mer de Glace (MDG), des Bossons (BOS) de Talèfre (TAL) et de Leschaux (LES).

2007) et d'un glacier népalais, le Mera Glacier, depuis 2007. L'observation satellitaire est complémentaire de ces études in situ puisqu'elle permet de caractériser l'évolution glaciaire à l'échelle régionale.

En comparant une topographie déduite d'images Spot 5 de novembre 2004 avec des mesures de la navette spatiale américaine (mission SRTM pour Shuttle Radar Topographic Mission) datant de février 2000, des fortes pertes d'épaisseur à basse altitude sont observées pour $915 \mathrm{~km}^{2}$ de glaciers de la région du Spiti et Lahaul dans l'Himalaya indien (figure 4), alors que, comme dans les Alpes, les zones les plus hautes restent stables. Entre février 2000 et novembre 2004, 3,9 $\mathrm{km}^{3}$ de glace ont disparu. Une fois divisées par la surface totale des glaciers, ces pertes correspondent à la disparition d'une lame d'eau de 0,7 à $0,8 \mathrm{~m}$ chaque année (Berthier et al., 2007). C'est considérable. Cette forte diminution de masse est confirmée par les mesures de terrain (Wagnon et al., 2007). Toutefois, notre étude couvre une trop courte période de temps et reste trop localisée pour conclure à un recul généralisé des glaciers himalayens. Cette chaîne de montagne, qui s'allonge sur $2500 \mathrm{~km}$ depuis le Bouthan à l'est jusqu'au

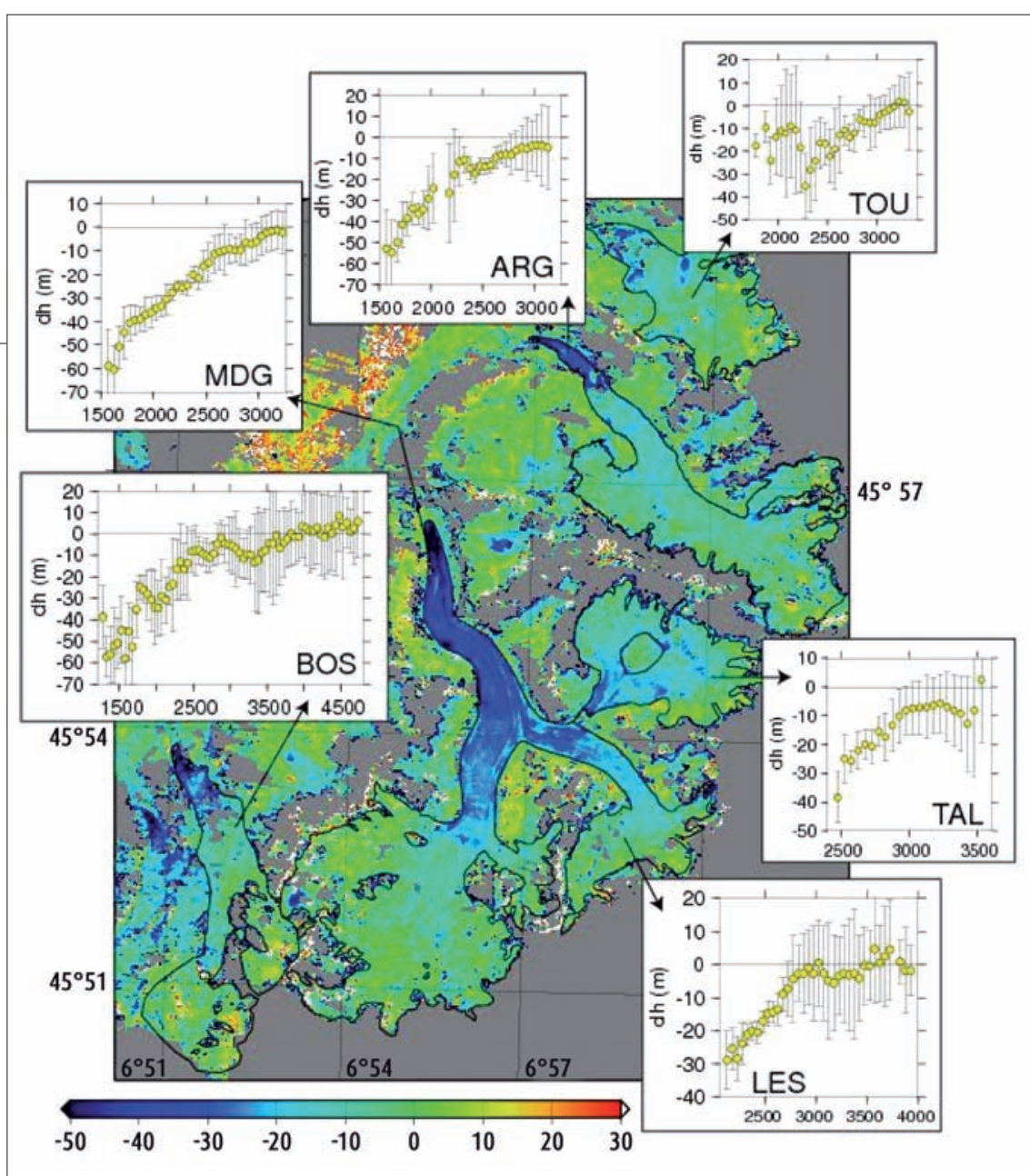

Karakorum à l'ouest, est soumise à des influences climatiques très différentes. C'est ainsi que l'essentiel des précipitations est amené par la mousson et tombe au cours de l'été dans la partie est de la chaîne alors que les pluies sont concentrées en hiver et dans un flux d'ouest pour le Karakorum. On peut donc s'attendre à des réponses très différentes des glaciers soumis à des influences climatiques aussi variées. C'est ce que nous allons étudier dans les années à venir en planifiant des

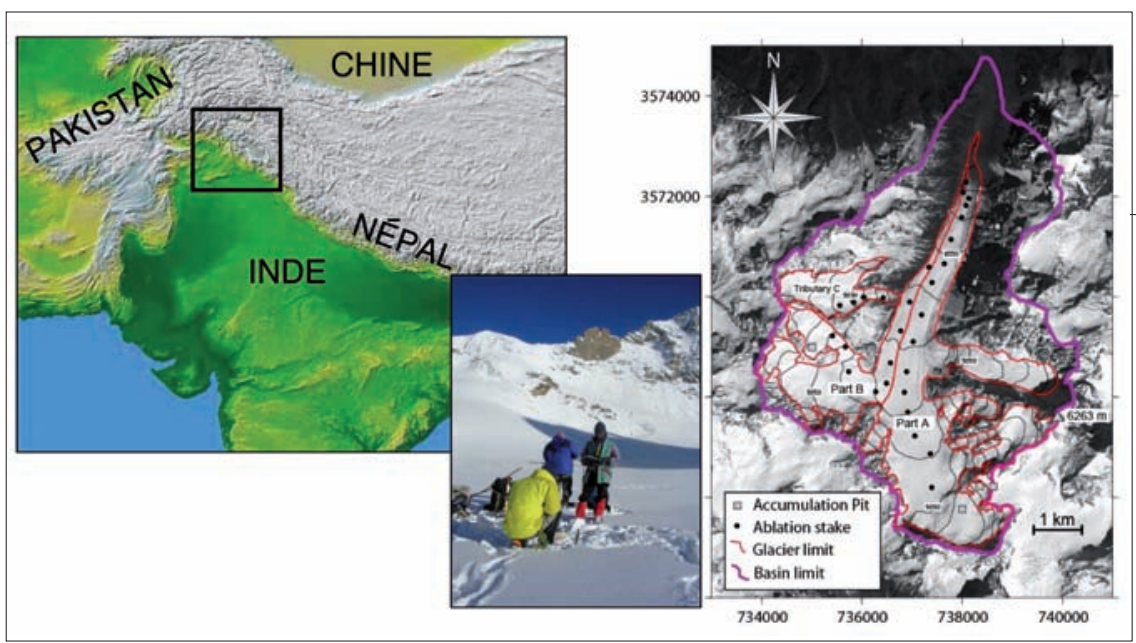

acquisitions satellitaires le long d'un transect est-ouest des chaînes du Karakorum et de l'Himalaya.

À ces mesures dans les Alpes et l'Himalaya, nous pouvons également ajouter des observations similaires que nous avons réalisées sur les glaciers islandais avec des collègues du Institute of Earth Science à Reykjavik. La similitude des pertes de masse dans les Alpes, en Islande ou dans l' Himalaya est étonnante pour des régions climatiquement si différentes (figure 5). Par leurs bilans de masse négatifs, ces trois régions contribueraient de façon significative $(+0,1 \mathrm{~mm} / \mathrm{an})$ à l'élévation du niveau moyen des mers depuis 1998-1999.

Figure 4 - Le glacier du Chhota Shigri dans l'ouest de la chaîne himalayenne.

À gauche, la topographie d'ensemble de la région. Au centre, photo de relevés de terrain dans la zone d'accumulation du glacier (C Yves Arnaud, IRD). À droite, une image Spot 5 du glacier du Chhota Shigri (novembre 2004) avec le réseau de balises et de puits d'accumulation qui permet d'estimer son bilan de masse sur le terrain.

En rouge, les limites du glacier; en bleu celles de son bassin versant; en noir, les courbes de niveau tous les 100 mètres.

(C) CNES 2004 / Distribution Spot Image) 
La comparaison des mesures satellitaires récentes à des relevés du Laboratoire de glaciologie dans les années 1965 à 1980 indique un ralentissement de 30 à $40 \%$ du mouvement de la langue de la mer de Glace. Nous tenons probablement ici l'explication à la partie manquante des pertes d'épaisseur dans la région basse de ce glacier : les flux de glaces de la partie haute vers la partie basse de la mer de Glace se réduisent et l'amincissement des zones basses est plus fort que celui attendu en réponse directe à l'augmentation des températures. Des calculs simples montrent en effet que cette décélération du glacier expliquerait près de la moitié de l'amincissement de la mer de Glace sous 2200 mètres. Ces chiffres sont préliminaires, mais ils démontrent l'importance d'un suivi détaillé de la dynamique.

La corrélation d'images permet donc de comprendre l'évolution des flux de glace, entre la zone d'accumulation et d'ablation, et le temps de réponse des glaciers dans un contexte de réchauffement global rapide. Mais cette technique est également adaptée pour l'observation des variations saisonnières des vitesses glaciaires. Ces variations sont importantes car elles sont contrôlées par les conditions hydrolo-

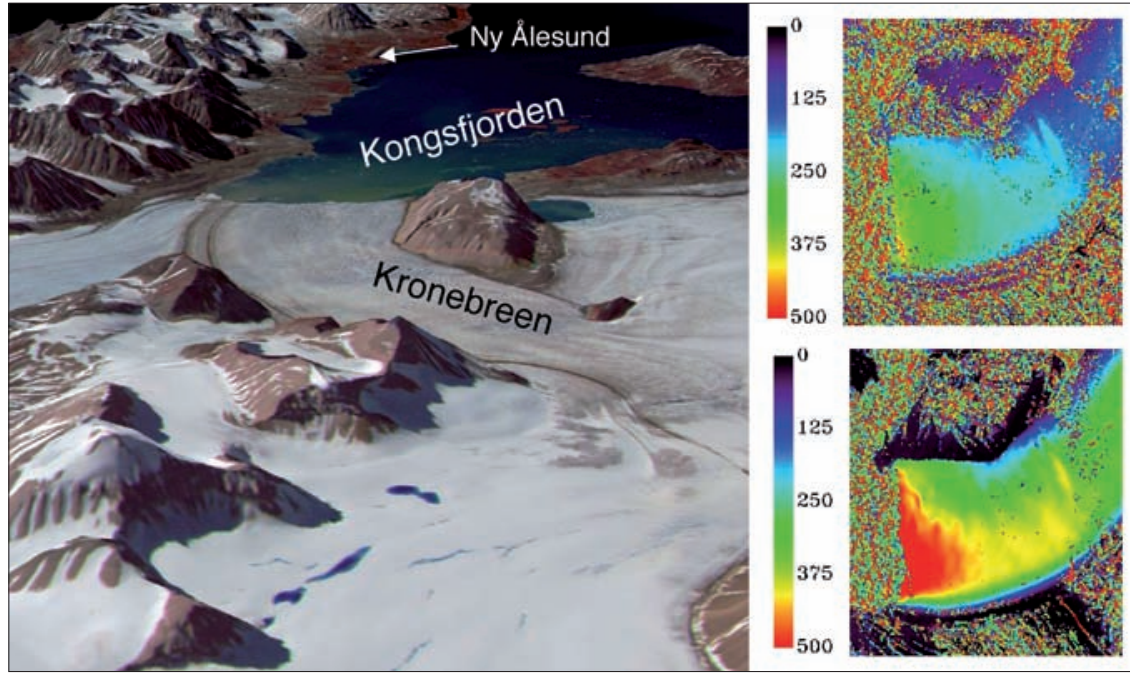

Figure 7 - Variations saisonnières des vitesses du glacier Kronebreen (Spitzberg). À gauche, vue 3D du fjord du Roi (le Kongsfjorden) à partir d'une image Aster (C) Nasa/Ersdac). À droite comparaison des vitesses d'écoulement du glacier (en mètres par an) au printemps (en haut, entre le $1^{\text {er }}$ mai et 14 juin 2007) et en été (en bas, entre le 26 juin et le 2 août 2007). La même échelle de couleur a été utilisée pour les deux champs de vitesse afin de mettre en évidence la forte accélération de ce glacier au début de l'été

giques sous-glaciaires, elles-mêmes dépendantes de l'intensité de la fonte en surface du glacier. Dans le cas de la calotte groenlandaise, on tient une explication possible (mais discutée !) de l'accélération récente de certains glaciers émissaires (Van de Wal et al., 2008). Dans le cadre de l'Année polaire internationale et grâce aux images d'un satellite taiwanais (Formosat-2), nous étudions de telles variations de vitesse pour le glacier Kronebreen, qui vêle ses icebergs dans le fjord du Roi au Spitzberg (figure 7).

La même technique de corrélation d'images Spot 5 a aussi été utilisée dans un contexte plus original pour étudier

Figure 8 - Le lac sous-glaciaire de Grimsvötn : un calorimètre sous la calotte glaciaire du Vatnajökull. La petite carte permet de situer le Vatnajökull au sud-est de I'Islande. (a) Image Landsat avec la localisation de la plate-forme au centre de la calotte glaciaire. (b) Photographie aérienne de l'éruption volcanique de début novembre 2004 (C M. T. Gudmundsson, Institute of Earth Science). (c) Fonctionnement du lac sous-glaciaire de Grimsvötn. En mesurant, depuis l'espace, le soulèvement de la plate-forme de glace flottant au-dessus du lac, on peut déduire le flux géothermique de cette région à forte activité volcanique.

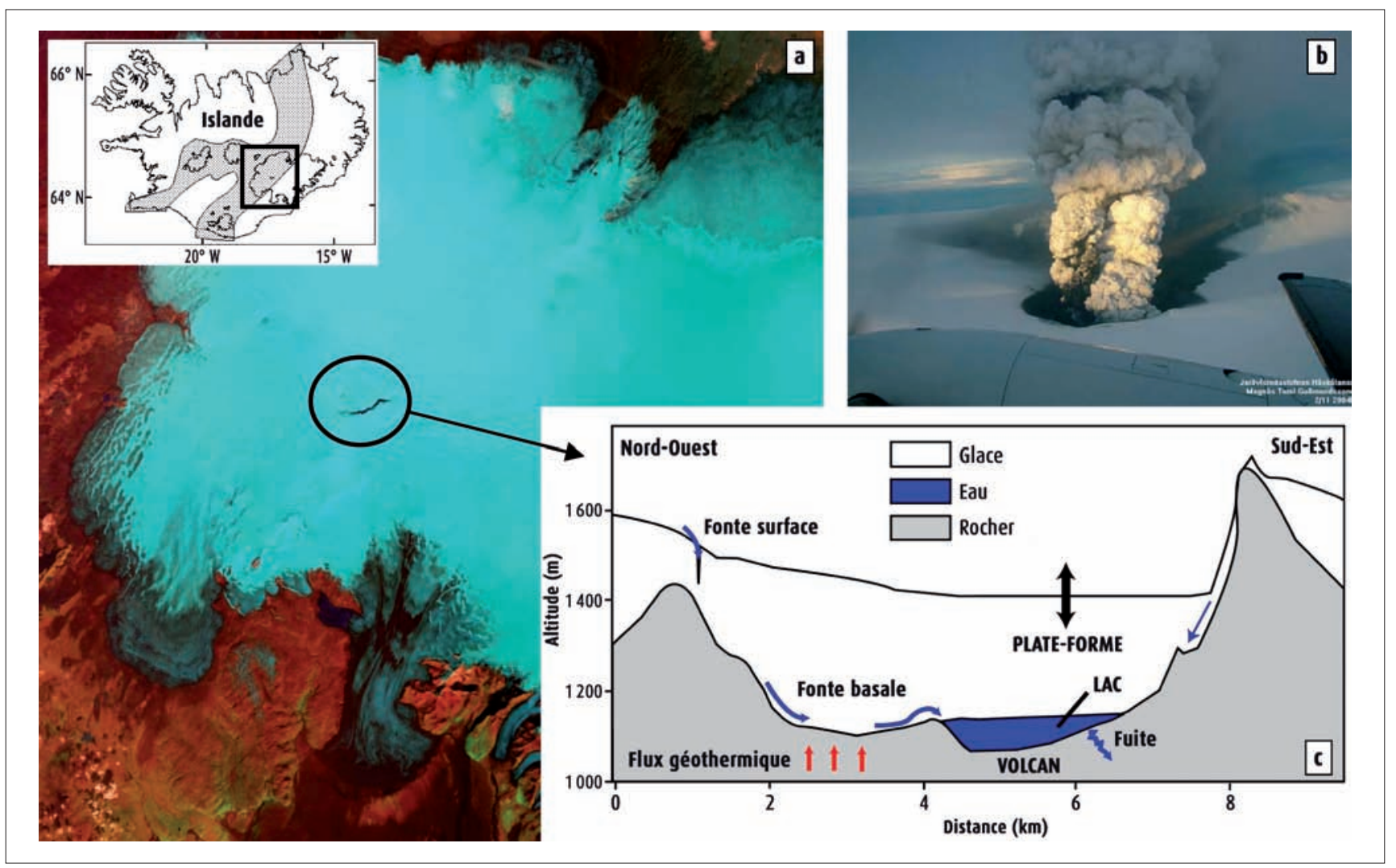


l'un des aspects de la dynamique de la calotte glaciaire du Vatnajökull (Islande). Cette fois, ce n'est pas le mouvement horizontal du glacier qui nous intéressait, mais ses mouvements verticaux. Nous avons donc corrélé deux images Spot 5 acquises à cinq jours d'intervalle en août 2004 avec des incidences très proches et obliques afin de détecter le mouvement d'une plate-forme de glace qui flotte au-dessus du lac sous-glaciaire de Grímsvötn (figure 8). À partir de la carte du soulèvement, nous avons pu délimiter l'extension du lac sous-glaciaire et, grâce à de simples principes de calorimétrie, estimer l'activité géothermique de cette zone située à l'aplomb d'un volcan actif (Berthier et al., 2006). Quelques semaines avant une éruption majeure en novembre 2004, nous avons ainsi déduit une activité géothermique particulièrement forte avec un flux de chaleur de 60 à $70 \mathrm{~W} / \mathrm{m}^{2}$.

\section{Observations satellitaires et Année polaire internationale}

En mars 2007 a été lancée la quatrième Année polaire internationale (API) qui suit celles qui se sont déroulées en 1882,1932 et 1957 . Elle durera jusqu'en mars 2009. L'un des projets phares de cette API, GIIPSY (http://bprc.osu.edu/rsl/ GIIPSY/), vise à coordonner les efforts des différentes agences spatiales (Nasa, ESA, CNES, Jaxa, etc.) afin d'obtenir une couverture complète des régions polaires avec des capteurs spatiaux variés (imagerie optique haute et basse résolution, radar à synthèse d'ouverture, altimétrie laser et radar...). Au Legos, nous sommes les responsables scientifiques de la contribution française à GIIPSY, le projet Spirit (Spot 5 Stereoscopic Survey of Polar Ice: Reference Images and Topographies ; www.spotimage. fr/IPY/). Spirit est le fruit d'une étroite collaboration avec Spot Image, qui met le capteur Spot 5-HRS (pour haute résolution stéréoscopique) au service des glaciologues. Ce projet est financé intégralement par le Cnes. Il s'agit d'acquérir une archive unique de couples d'images stéréoscopiques couvrant toute la périphérie de l'Antarctique, du Groenland et l'ensemble des autres masses de glaces polaires (Alaska, Patagonie, Islande, Spitzberg...). Le capteur HRS allie une haute résolution (environ $10 \mathrm{~m}$ ) à une large couverture spatiale puisque la fauchée est de $120 \mathrm{~km}$ le long de segments couvrant jusqu'à 600 km (figure 9). IGN Espace est associée au projet afin de déduire de ces images une topographie de référence qui manque cruellement pour l'étude des glaces polaires.

Une autre agence spatiale a aussi répondu présent aux sollicitations des glaciologues pour l'Année polaire internationale, l'agence spatiale taiwanaise (NSPO). Le NSPO a débuté au cours de l'été 2007 des acquisitions Formosat-2, un satellite récent qui allie une haute résolution géométrique ( $2 \mathrm{~m}$ en mode panchromatique) à une bonne capacité de revisite (une image utile environ chaque semaine dans le contexte nuageux de la haute montagne). Ces acquisitions vont permettre une description fine de la variabilité spatiale et temporelle des vitesses des écoulements glaciaires, notamment dans le cas des larges glaciers émissaires des calottes polaires. Nous avons montré plus haut des premiers résultats sur le Spitzberg (figure 7). Ces images Formosat-2 pourraient aussi nous permettre de suivre l'activité des lacs sous-glaciaires antarctiques en observant les mouvements verticaux de la glace qui les recouvre comme nous l'avons déjà fait pour la calotte du Vatnajökull.

\section{Conclusion}

À partir d'images satellitaires optiques à haute résolution et de traitements adaptés au contexte glaciaire, nous pouvons désormais caractériser à l'échelle régionale l'impact des changements climatiques sur le volume et la dynamique des glaciers. Dans l'avenir, ces mêmes outils devraient contribuer à obtenir une vision globale de l'évolution des masses de glace terrestre et, notamment, des calottes des régions polaires.

La télédétection est donc devenue un outil clé pour suivre l'évolution des glaces tempérées et polaires. Elle est complémentaire des mesures de terrain qui restent indispensables pour valider les mesures satellitaires et, aussi, parce que certains paramètres ne peuvent tout simplement pas être mesurés depuis l'espace. Nos travaux en imagerie optique sont possibles grâce à l'apport récent de la haute résolution (de l'ordre de quelques mètres). Dans l'avenir proche, les images optiques à très haute résolution (résolution inférieure au mètre) seront utiles pour étudier encore plus précisément l'évolution de glaciers

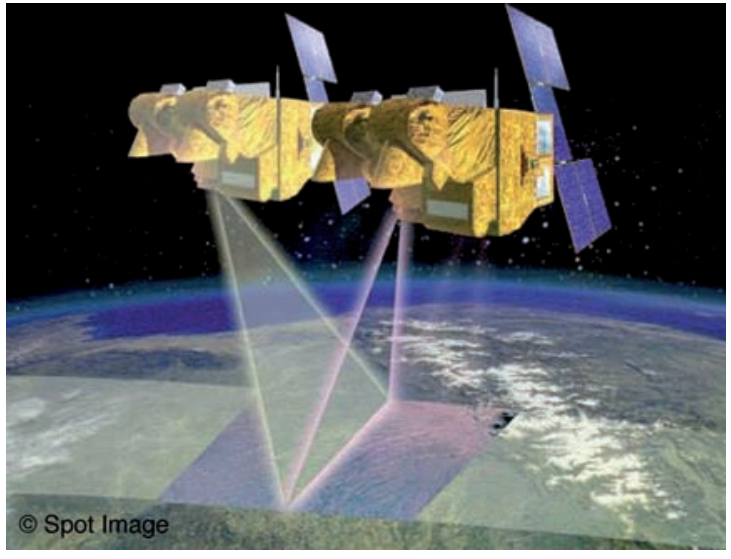

Figure 9 - Vue d'artiste du capteur Spot 5-HRS. (C) Spot Image)

de petite taille. En revanche, ces images couvriront des zones géographiques beaucoup plus réduites, ce qui sera préjudiciable au suivi des plus grands glaciers. Par exemple, la fauchée des satellites de la future constellation Pleiades sera de $20 \mathrm{~km}$ contre $60 \mathrm{~km}$ pour ceux de la famille Spot. À ce titre, et malgré un satellite très sollicité (donc des difficultés pour obtenir des images...), la mission Spot 5 (capteurs HRG et surtout HRS) est sans doute la plus attrayante pour la communauté des glaciologues. Par conséquent, au moment où l'intérêt des scientifiques, de la société civile et des médias va grandissant autour de l'évolution des glaciers et du climat des régions polaires, il nous semble urgent d'envisager aujourd'hui un successeur à Spot 5.

\section{Remerciements}

Avant tout, je tiens à remercier pour leur soutien mes deux directeurs de thèse, Frédérique Rémy (CNRS-Legos) et Yves Arnaud (IRD-LTHE). Les discussions et les observations de terrain fournies par (ou acquises avec) Christian Vincent (CNRS-LGGE) ainsi que Helgi Björnsson, Finnur Pálsson et Sverrir Guðmundsson (de l'université d'Islande) et Garry Clarke (de l'université de Colombie-Britannique) ont été un des éléments clés de ce travail. La relecture par deux rapporteurs a permis de nettement améliorer la qualité de ce manuscrit. Merci au comité de rédaction de La Météorologie. Ces travaux ont été financés par une bourse de doctorat du ministère de l'Éducation nationale, par l'ACI Observation de la Terre, une bourse postdoctorale européenne Marie Curie et par le Tosca (projet TOP Glace API). Nos études n'auraient pas été possibles sans les images Spot obtenues dans le cadre du programme Isis du Cnes (http://medias.obs-mip. fr/isis2/). 


\section{Bibliographie}

\footnotetext{
Arendt A. A., K. A Echelmeyer, W. D. Harrison, C. S. Lingle et V. B. Valentine, 2002 : Rapid wastage of Alaska glaciers and their contribution to rising sea level. - Science, 297(5580), 382-386.

- Berthier E., Y. Arnaud, D. Baratoux, C. Vincent et F. Remy, 2004 : Recent rapid thinning of the "Mer de Glace" glacier derived from satellite optical images. Geophys. Res. Lett., 31(17), L17401.

- Berthier E., Y. Arnaud, R. Kumar, S. Ahmad, P. Wagnon et P. Chevallier, 2007 : Remote sensing estimates of glacier mass balances in the Himachal Pradesh - (Western Himalaya, India). Remote Sensing of Environment, 108(3), 327-338.

Berthier E., H. Björnsson, F. Palsson, K. L. Feigl, M. Llubes et F. Rémy, 2006 : The level of the Grimsvotn subglacial lake, Vatnajokull, Iceland, monitored with SP0T5 - images. Earth and Planetary Science Letters, 243(1-2), 293-302.

- Berthier E., H. Vadon, D. Baratoux, Y. Arnaud, C. Vincent, K. L. Feigl, F. Rémy et B. Legresy, 2005 : Surface motion of mountain glaciers derived from satellite opti- cal imagery. Remote Sensing of Environment, 95(1), 14-28.

- Braithwaite R. J., 2002 : Glacier mass balance: the first 50 years of international monitoring. Progress in Physical Geography, $26(1), 76-95$.

- Cazenave A., K. DoMinh, S. Guinehut, E. Berthier, W. Llovel, G. Ramillien, M. Ablain et G. Larnicol, (Sous presse). Sea level budget over 2003-2008: a reeva-

- luation from GRACE space gravimetry, satellite altimetry and Argo. Global and Planetary Change.

- Dyurgerov M. B. et M. F. Meier, 2005 : Glaciers and the Changing Earth System: a 2004 Snapshot. Boulder, Instaar.

- Fischer A., H. Rott et H. Björnsson, 2003 : Observation of recent surges of Vatnajokull, Iceland, by means of ERS SAR interferometry. Annals of Glaciology, $37,69-76$.

- Francou B. et C. Vincent, 2007 : Les glaciers à l'épreuve du climat : Belin, IRD.

- IPCC International Panel on Climate Change, 2007 : Climate Change 2007: The Scientific Basis. New York, Cambridge Univ. Press.

Kaser G., J. G. Cogley, M. B. Dyurgerov, M. F. Meier et A. Ohmura, 2006 : Mass balance of glaciers and ice caps: Consensus estimates for 1961-2004. Geophys. Res. Lett., 33(19), L19501.

- Meier M. F., 1984 : Contribution of Small Glaciers to Global Sea Level. Science, 226(4681), 1418-1421.

- Oerlemans J., 2005 : Extracting a climate signal from 169 glacier records. Science, 308(5722), 675-677.

- Ohmura A., A. Bauder, H. Müller et G. Kappenberger, 2007 : Long-Term change of mass balance and th role of radiation. Annals of Glaciology, $46,367-374$.

- Paul F., A. Kääb, M. Maisch, T. Kellenberger et W. Haeberli, 2002 : The new remote-sensing-derived swiss glacier inventory: I. Methods. Annals of Glaciology, 34, 355-361.

Rémy F. et M. Frezzotti, 2006 : Antarctica ice sheet mass balance. Comptes Rendus Geoscience, 338(14-15), 1084-1097.

- Rémy F., L. Testut et B. Legresy, 2000 : Ice sheet topography derived from satellite altimetry. Comptes rendus de l'Académie des sciences. Série 2. Sciences de la terre et des planètes, 330(7), 457-467.

- Scambos T., M. J. Dutkiewicz, J. C. Wilson et R. A. Bindschadler, 1992 : Application of image cross-correlation to the measurement of glacial velocity using satel- lite image data. Remote Sensing of Environment, 42, 177-186.

- Vallon M., 1977 : Bilan de masse et fluctuations récentes du glacier Ampère (îles Kerguelen, TAAF). Zeitschrift für Gletscherkunde und Glazialgeologie, $13,55-85$.

- Van de Wal R. S. W., W. Boot, M. R. Van den Broecke, C. J. P. P. Smeets, C. H. Reijmer, J. J. A. Donker et J. Oerlemans, 2008 : Large and rapid melt- induced velocity changes in the ablation zone of the Greenland lce Sheet. Science, 321, 111-113.

- Vincent C., G. Kappenberger, F. Valla, A. Bauder, M. Funk et E. Le Meur, 2004 : Ice ablation as evidence of climate change in the Alps over the 20 th century. Journal of Geophysical Research-Atmospheres, 109, (D10) 1-9.

Vincent C., E. Le Meur, D. Six, M. Funk, M. Hoelzle et S. Preunkert, 2007a : Very high-elevation Mont Blanc glaciated areas not affected by the 20th Century climate change. J. Geophys. Res., 112(D9), D09120.

- Vincent C., E. Le Meur, D. Six et E. Thibert, 2007b : Un service d'observation des glaciers des Alpes françaises " glacioclim-alpes ", pour quoi faire ? La Houille Blanche, 03, 86-95.

- Wagnon P., K. Rajesh, Y. Arnaud, A. Linda, P. Sharma, C. Vincent, J. Pottakal, E. Berthier, A. Ramanathan, S. I. Hasnain et P. Chevallier, 2007 : Four - years of mass balance on Chhota Shigri Glacier (Himachal Pradesh, India), a new benchmark glacier in the Western Himalaya. Journal of Glaciology, 53(183), 603-611.
} 